\title{
A new method of generating fuzzy implications from given ones
}

\author{
S. Massanet ${ }^{1}$ J. Torrens ${ }^{1}$ \\ ${ }^{1}$ Dept. of Math. and Comp. Science, University of the Balearic Islands, 07122 Palma de Mallorca, Spain
}

\begin{abstract}
In this paper, a new construction method of a fuzzy implication from two given ones, called e-generation method, is introduced. This method allows to control, up to a certain level, the increasingness on the second variable of the fuzzy implication through an adequate scaling on that variable of the two given implications. The natural propagation of the most usual properties of fuzzy implications from the initial ones to the constructed implication is studied. Unlike majority of existing construction methods of fuzzy implications, this model preserves the exchange principle, even the law of importation, under certain conditions.
\end{abstract}

Keywords: Fuzzy implication, e-generation method, contrapositive symmetry, exchange principle, law of importation.

\section{Introduction}

Fuzzy implications, the generalization of the classical implications to the multi-valued framework, have probably become the most important operations in fuzzy logic, approximate reasoning and fuzzy control. These operators are not limited to model fuzzy conditionals, but also to make inferences in any fuzzy rule based system ([1], [2]). However, the fact that many authors have focused their interest in the theoretical study of fuzzy implications as highlight the surveys [3] and [4], and also the recent book [5], exclusively devoted to fuzzy implications is based on a wider range of applications. Thus, fuzzy implications are useful in fuzzy relational equations and fuzzy mathematical morphology ([3]), fuzzy DI-subsethood measures and image processing ([6], [7]), data mining ([8]), computing with words ([3]), fuzzy equivalence relations and fuzzy partitions ([9]).

All these properties emphasize the necessity of having a large repertoire of fuzzy implications at one's disposal. There are many different models of fuzzy implications and more than forty implications have been used just in fuzzy control. In [10], the meaning and the behaviour of a model of fuzzy implications is pointed out as the crucial point to its election. In this way, there are some methods in order to generate a new fuzzy implication from two given ones. Among them, the following ones are the most remarkable (Chapter 6, [5]):

- The min and max operations generate new fuzzy implications from two given ones $I, J$ :

$$
\begin{aligned}
& (I \vee J)(x, y)=\max \{I(x, y), J(x, y)\}, x, y \in[0,1], \\
& (I \wedge J)(x, y)=\min \{I(x, y), J(x, y)\}, x, y \in[0,1] .
\end{aligned}
$$

- The convex combinations of fuzzy implications also provides new fuzzy implications from two given ones $I, J$ and $\lambda \in \mathbb{R}$ :

$$
K(x, y)=\lambda \cdot I(x, y)+(1-\lambda) \cdot J(x, y), \quad x, y \in[0,1] .
$$

- Given a t-norm $T$, the composition of fuzzy implications $I, J$ is defined as follows

$$
(I \stackrel{T}{\circ} J)(x, z)=\sup _{y \in[0,1]}(T(I(x, y), J(y, z))),
$$

with $x, z \in[0,1]$. However, this method only generates a fuzzy implication if and only if $(I \circ$ $J)(1,0)=0$ (Theorem 6.4.4. in [5]).

In addition, from an existing implication $I$ and an automorphism $\varphi$, the $\varphi$-conjugation of $I$ is defined as

$$
I_{\varphi}(x, y)=\varphi^{-1}(I(\varphi(x), \varphi(y))), \quad x, y \in[0,1] .
$$

The importance of these generation methods is related to the preservation of the properties of the initial implications to the final one. Although all the methods preserve certain properties, only the conjugacy preserves the exchange principle, one of the most important properties of the fuzzy implications. Thus, the current generation methods from two implications do not ensure a resulting implication satisfying the exchange principle even when the two initial ones satisfy it.

The generation method presented in this paper, egeneration method of a fuzzy implication, preserves the exchange principle and many other properties. When a property is not always preserved, the necessary conditions to ensure that this fact happens are studied. In addition, it allows to have a degree of control of the increasingness respect to the second variable of the fuzzy implication. Thus, given an $e \in(0,1)$, the new implication is equal to or greater than $e$ above $e$ and equal to or less than $e$ below $e$. 
This property is reminiscent of a new class of fuzzy implications generated from additive generators of representable uninorms, called $h$-implications (see [11]). The communication is organized as follows. In the next section we recall the basic definitions and properties of implications needed in the subsequent sections. In Section 3, we present the egeneration method of a fuzzy implication from two given ones and we discuss then, the basic properties preserved by this new method. In Section 4, the contrapositive symmetry is deeply studied as well as the exchange principle and the law of importation in Section 5. The paper ends with some conclusions and future work.

\section{Preliminaries}

We will suppose the reader to be familiar with the theory of t-norms and t-conorms (all necessary results and notations can be found in [12]). To make this work self-contained, we recall here some of the concepts and results employed in the rest of the paper.

\subsection{Fuzzy Negations}

Definition 1 (see Definition 1.1 in [13] and Definition 11.3 in [12]) $A$ decreasing function $N$ : $[0,1] \rightarrow[0,1]$ is called a fuzzy negation, if $N(0)=1$, $N(1)=0$. A fuzzy negation $N$ is called

(i). strict, if it is strictly decreasing and continuous.

(ii). strong, if it is an involution, i.e., $N(N(x))=x$ for all $x \in[0,1]$.

Example 2 Important negations that will be used along this paper are the classical fuzzy negation, $N_{C}(x)=1-x$, and the least fuzzy negation,

$$
N_{D_{1}}(x)= \begin{cases}1 & \text { if } x=0, \\ 0 & \text { if } x \in(0,1] .\end{cases}
$$

\subsection{Fuzzy Implications}

Definition 3 (see Definition 1.15 in [13], Definition 1.1 .1 in [5]) A binary operator $I:[0,1]^{2} \rightarrow$ $[0,1]$ is said to be an implication function, or an implication, if it satisfies:

(I1) $I(x, z) \geq I(y, z)$ when $x \leq y$, for all $z \in$ $[0,1]$.

(I2) $I(x, y) \leq I(x, z)$ when $y \leq z$, for all $x \in$ $[0,1]$.

(I3) $I(0,0)=I(1,1)=1$ and $I(1,0)=0$.

Example 4 In addition of the basic fuzzy implications, that are collected in Table 1.3. in [5], special implications that will be used along this paper are the least fuzzy implication

$$
I_{L t}(x, y)= \begin{cases}1 & \text { if } x=0 \text { or } y=1 \\ 0 & \text { if } x>0 \text { and } y<1\end{cases}
$$

and the greatest fuzzy implication

$$
I_{G t}(x, y)= \begin{cases}1 & \text { if } x<1 \text { or } y>0, \\ 0 & \text { if } x=1 \text { and } y=0\end{cases}
$$

Note that, from the definition, it follows that $I(0, x)=1$ and $I(x, 1)=1$ for all $x \in[0,1]$ whereas the symmetrical values $I(x, 0)$ and $I(1, x)$ are not derived from the definition. We will denote by $\mathcal{F} \mathcal{I}$ the set of all implications. Special interesting properties for implication functions are:

- The exchange principle,

$$
I(x, I(y, z))=I(y, I(x, z)), \quad x, y, z \in[0,1] .
$$

- The law of importation with a t-norm $T$,

$$
I(T(x, y), z)=I(x, I(y, z)), \quad x, y, z \in[0,1] .
$$

- The weak law of importation with a conjunctive, commutative and non-decreasing function $F:[0,1]^{2} \rightarrow[0,1],($ see $[14])$

$$
I(F(x, y), z)=I(x, I(y, z)), \quad x, y, z \in[0,1] .
$$

(WLI)

- The left neutrality principle,

$$
I(1, y)=y, \quad y \in[0,1] .
$$

- The ordering property,

$$
x \leq y \Longleftrightarrow I(x, y)=1, \quad x, y \in[0,1] .
$$

- The identity principle,

$$
I(x, x)=1, \quad x \in[0,1] .
$$

- The contrapositive symmetry with respect to a fuzzy negation $N$,

$$
I(x, y)=I(N(y), N(x)), \quad x, y \in[0,1] .
$$

Definition 5 (see Definition 1.14.15 in [5]) Let I be a fuzzy implication. The function $N_{I}$ defined by $N_{I}(x)=I(x, 0)$ for all $x \in[0,1]$, is called the natural negation of $I$.

\section{3. e-generation Method of a Fuzzy Implication}

The e-generation method of a fuzzy implication from two given ones is based on an adequate scaling on the second variable of the initial implications. The interesting property of the controlled increasingness is an straightforward consequence of the definition of the method. Note that the scaled first implication $I_{1}$ generates the new implication for $y \leq e$ and the second one, $I_{2}$ for $y>e$.

Theorem 6 Let $I_{1}, I_{2}$ be two implications and $e \in$ $(0,1)$. Then the binary function $I_{I_{1}-I_{2}}:[0,1]^{2} \rightarrow$ 
$[0,1]$, called the e-generated implication from $I_{1}$ and $I_{2}$, defined as $I_{I_{1}-I_{2}}(x, y)=$

$$
= \begin{cases}1 & \text { if } x=0, \\ e \cdot I_{1}\left(x, \frac{y}{e}\right) & \text { if } x>0, y \leq e, \\ e+(1-e) \cdot I_{2}\left(x, \frac{y-e}{1-e}\right) & \text { if } x>0, y>e,\end{cases}
$$

is a fuzzy implication.

Example 7 Let us see some of the e-generated implications from some well-known fuzzy implications (see Table 1.3 in [5]). The plot of these implications can be viewed in Figure 1 and Figure 2.

(i) If we consider the Eukasiewicz implication twice and $e=\frac{1}{2}$, we obtain the following $e$ generated implication $I_{I_{L K}-I_{L K}}(x, y)=$

$$
= \begin{cases}1 & \text { if } x=0 \\ \min \left\{\frac{1}{2}, \frac{1}{2}-\frac{x}{2}+y\right\} & \text { if } x>0 \text { and } y \leq \frac{1}{2} \\ \min \left\{1, \frac{1}{2}-\frac{x}{2}+y\right\} & \text { if } x>0 \text { and } y>\frac{1}{2}\end{cases}
$$

(ii) Let us consider the Kleene-Dienes implication, the Rescher implication and $e=\frac{1}{3}$. The following e-generated implication is obtained $I_{I_{K D}-I_{R S}}(x, y)=$

$$
= \begin{cases}1 & \text { if } x=0, \\ \max \left\{\frac{1-x}{3}, y\right\} & \text { if } x>0 \text { and } y \leq \frac{1}{3}, \\ \frac{1}{3} & \text { if } y>\frac{1}{3} \text { and } x>\frac{3 y-1}{2}, \\ 1 & \text { if } y>\frac{1}{3} \text { and } 0<x \leq \frac{3 y-1}{2} .\end{cases}
$$

(iii) Taking the Weber implication, the Reichenbach implication and $e \in(0,1)$, we obtain the following family of e-generated implications $I_{I_{W B}-I_{R C}}(x, y)=$

$$
= \begin{cases}1 & \text { if } x=0, \\ e & \text { if } 0<x<1, y \leq e, \\ y & \text { if } x=1, y \leq e, \\ e+(1-e)\left(1-x+x \cdot \frac{y-e}{1-e}\right) & \text { if } x>0, y>e .\end{cases}
$$

\subsection{Basic Properties}

Assuming that the initial implications $I_{1}, I_{2}$ satisfy a given property, we want to study in this section when such property is preserved by the $e$-generation method. When the property is not straightforward preserved, we study which conditions must satisfy the initial implications in order to ensure the preservation of the property. First of all, the already mentioned controlled increasingness on the second variable is fully determined.

Proposition 8 Let $I_{1}$ and $I_{2}$ be two implications. Then

(i) $I_{I_{1}-I_{2}}(x, y) \leq e$ if $x>0$ and $y<e$, $I_{I_{1}-I_{2}}(x, e)=e$ if $x>0$, $I_{I_{1}-I_{2}}(x, y) \geq e$ if $x>0$ and $y>e$.

(ii) When $x>0$ and $y<e$ then $I_{I_{1}-I_{2}}(x, y)<e \Leftrightarrow I_{1}(a, b)<1$ for $a>0, b<1$.

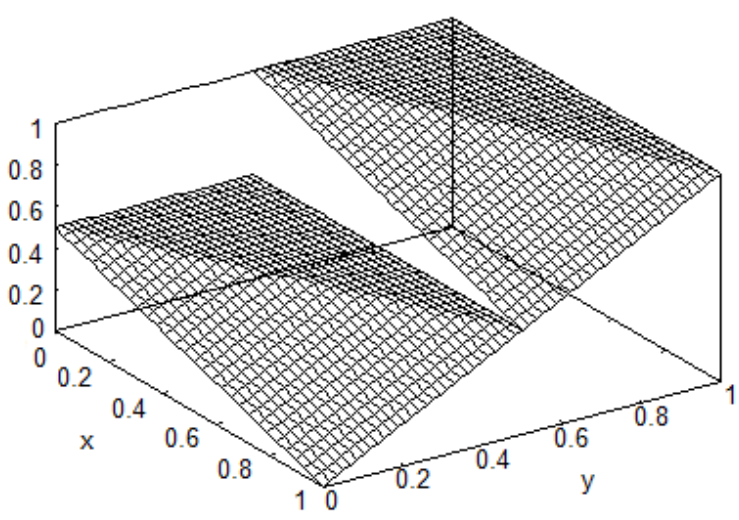

(a) $I_{I_{L K}-I_{L K}}$ with $e=\frac{1}{2}$

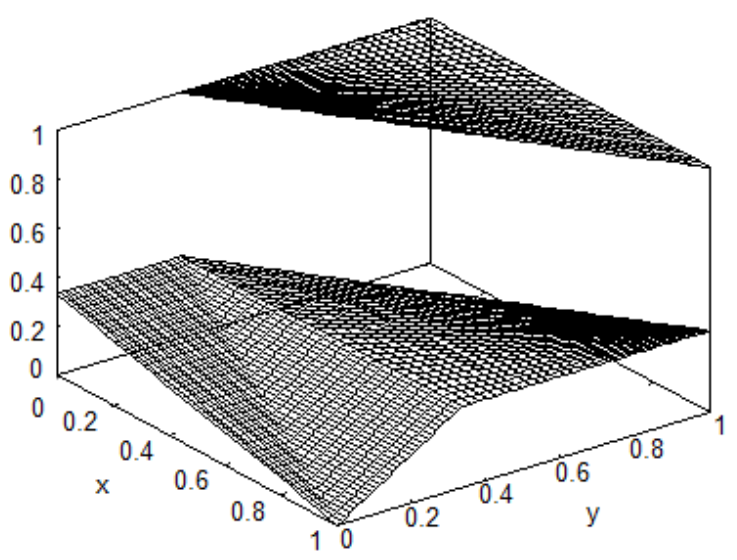

(b) $I_{I_{K D}-I_{R S}}$ with $e=\frac{1}{3}$

Figure 1: Plots of some e-generated implications

(iii) When $x>0$ and $y>e$ then $I_{I_{1}-I_{2}}(x, y)>e \Leftrightarrow I_{2}(a, b)>0$ for $a, b>0$.

The left neutrality property (NP) and the left $e$ neutrality property $\left(\mathrm{NP}_{e}\right)$, that is $I(e, y)=y$ for all $y \in[0,1]$ and for some fixed $e \in(0,1)$, are straightforward preserved.

Proposition 9 Let $I_{1}, I_{2}$ be two implications and $e \in(0,1)$. Then

$$
I_{I_{1}-I_{2}} \text { satisfies }(N P) \Leftrightarrow I_{1} \text { and } I_{2} \text { satisfy }(N P) \text {. }
$$$$
I_{I_{1}-I_{2}} \text { satisfies }\left(N P_{e}\right) \Leftrightarrow I_{1} \text { and } I_{2} \text { satisfy }\left(N P_{e}\right) \text {. }
$$

The natural negation of the e-generated implication is only generated by the natural negation of $I_{1}$ and it is never continuous.

Proposition 10 Let $I_{1}$ and $I_{2}$ be two implications. Then the natural negation of $I_{I_{1}-I_{2}}$ is

$$
N_{I_{I_{1}-I_{2}}}(x)= \begin{cases}1 & \text { if } x=0, \\ e \cdot N_{I_{1}}(x) & \text { if } x>0,\end{cases}
$$

which is always a non-continuous negation.

Corollary 11 Let $I_{1}$ and $I_{2}$ be two implications. Then

$$
N_{I_{I_{1}-I_{2}}}=N_{D_{1}} \Leftrightarrow N_{I_{1}}=N_{D_{1}} \text {. }
$$




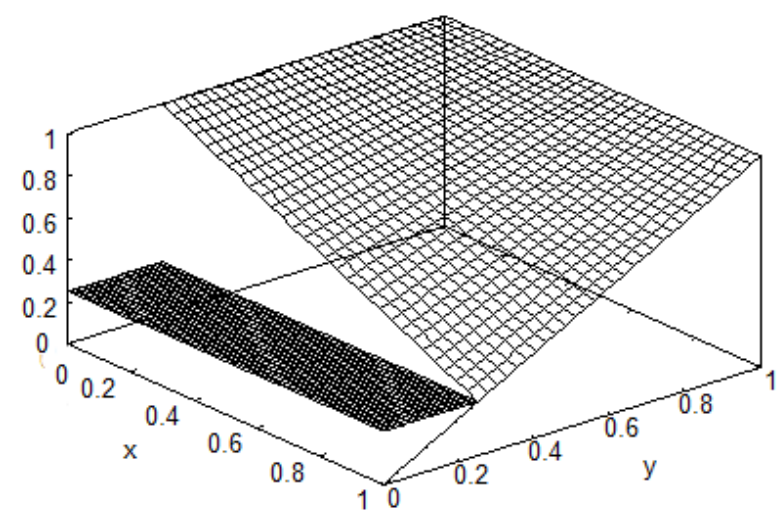

(a) $I_{I_{W B}-I_{R C}}$ with $e=\frac{1}{4}$

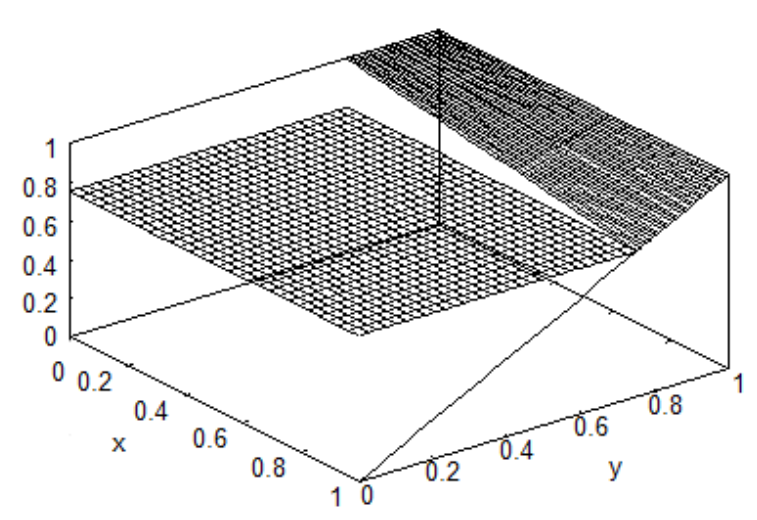

(b) $I_{I_{W B}-I_{R C}}$ with $e=\frac{3}{4}$

Figure 2: Plots of some e-generated implications from $I_{W B}$ and $I_{R C}$

The two following results show that while (IP) is never satisfied by an e-generated implication, the $e$ identity principle $\left(\mathrm{IP}_{e}\right)$, that is $I(x, x)=e$ for all $x \in(0,1)$ and for some fixed $e \in(0,1)$, is satisfied in some cases.

Proposition 12 Let $I_{1}$ and $I_{2}$ be two implications. Then $I_{I_{1}-I_{2}}(x, x)=1$ if and only if $x=0$ or $x>$ e with $I_{2}\left(x, \frac{x-e}{1-e}\right)=1$, i.e., $I_{I_{1}-I_{2}}$ does not satisfy (IP).

Proposition 13 Let $I_{1}, I_{2}$ be two implications and $e \in(0,1)$. Then $I_{I_{1}-I_{2}}$ satisfies $\left(I P_{e}\right)$ if and only if $I_{1}\left(x, \frac{x}{e}\right)=1$ for all $0<x \leq e$ and $I_{2}\left(x, \frac{x-e}{1-e}\right)=0$ for all $e<x<1$.

The following example shows an e-generated implication satisfying $\left(\mathrm{IP}_{e}\right)$ although neither $I_{1}$ nor $I_{2}$ satisfy it.

Example 14 Let $I_{G D}$ be the Gödel implication and $I_{R S}$ the Rescher implication and $e \in(0,1)$ then $I_{I_{G D}-I_{R S}}(x, y)=$

$$
=\left\{\begin{array}{l}
1 \quad \text { if } x=0 \text { or } x>0, y>e, x \leq \frac{y-e}{1-e}, \\
y \quad \text { if } x>0, y \leq e, x>\frac{y}{e} \\
e \quad \text { otherwise }
\end{array}\right.
$$

is an e-generated implication satisfying $\left(I P_{e}\right)$, that can be viewed in Figure 3.

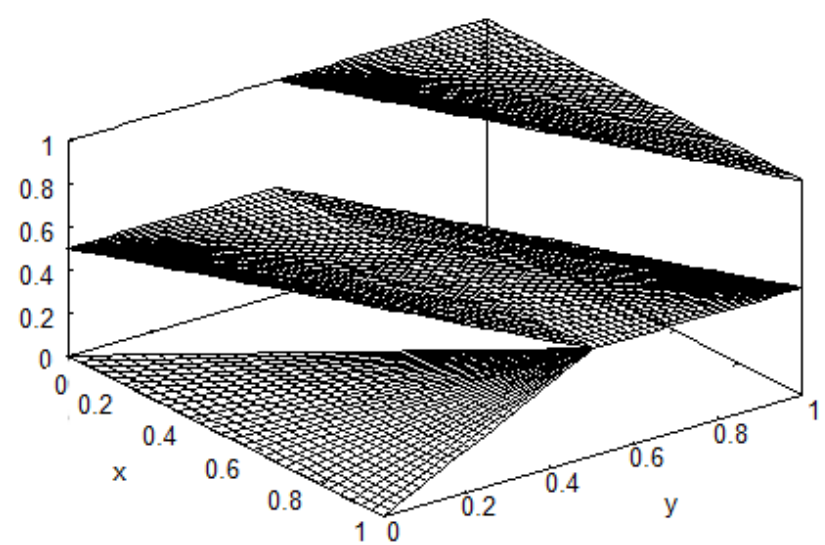

Figure 3: Plot of the $I_{I_{G D}-I_{R S}}$, an e-generated implication satisfying $\left(\mathrm{IP}_{e}\right)$ with $e=\frac{1}{2}$.

On the other hand, an e-generated implication does not satisfy (OP) nor the $e$-ordering property $\left(\mathrm{OP}_{e}\right)$, that is $I(x, y) \geq e \Leftrightarrow x \leq y$ for some fixed $e \in(0,1)$.

Proposition 15 Let $I_{1}$ and $I_{2}$ be two implications. Then $I_{I_{1}-I_{2}}(x, y)=1$ if and only if $x=0$ or $(x>0$ and $y>e$ ) with $I_{2}\left(x, \frac{y-e}{1-e}\right)=1$, i.e., $I_{I_{1}-I_{2}}$ does not satisfy $(O P)$.

Proposition 16 Let $I_{1}, I_{2}$ be two implications and $e \in(0,1)$. Then $I_{I_{1}-I_{2}}(x, y) \geq e$ if and only if $x=0$ or $(x>0$ and $y>e)$ or $(x>0$ and $y<e$ with $\left.I_{1}\left(x, \frac{y}{e}\right)=1\right)$, i.e., $I_{I_{1}-I_{2}}$ does not satisfy $\left(O P_{e}\right)$.

Finally, the next results show that the egenerated implication is never continuous but under some assumptions we can delimit the points of discontinuity.

Proposition 17 Let $I_{1}, I_{2}$ be two implications. Then $I_{I_{1}-I_{2}}$ is not continuous at the points $(0, y)$ with $y \leq e$.

Proposition 18 Let $I_{1}, I_{2}$ be two implications. Then $I_{I_{1}-I_{2}}$ is continuous everywhere except at the points $(0, y)$ with $y \leq e$ if and only if, $I_{1}$ is continuous everywhere except maybe at the points $(0, y)$ for all $y \in[0,1], I_{2}$ is continuous everywhere except at $(0,0)$ and $N_{I_{2}}=N_{D_{1}}$.

\section{Contrapositive Symmetry}

The contrapositive symmetry is one of the most studied properties that can be satisfied by an implication $I$ and a fuzzy negation $N$. This property is not straightforward preserved by the e-generation method (similarly to other generation methods). The e-generated implications satisfying $(\mathrm{CP}(\mathrm{N}))$ are not fully characterized, but some interesting results can be obtained. First of all, the following examples show that there exist e-generated implications that 
do not satisfy $(\mathrm{CP}(\mathrm{N}))$ for any negation $N$ and others that satisfy $(\mathrm{CP}(\mathrm{N}))$ for an infinity of negations $N$.

Example 19 (i) The e-generated implication $I_{I_{K D}-I_{K D}}(x, y)=$ $= \begin{cases}1 & \text { if } x=0, \\ \max \{e(1-x), y\} & \text { if } x>0 \text { and } y \leq e, \\ \max \{1-x+e x, y\} & \text { if } x>0 \text { and } y>e,\end{cases}$ where $I_{K D}$ is the Kleene-Dienes implication, does not satisfy $(C P(N))$ for any negation $N$, although $I_{K D}$ satisfies $\left(C P\left(N_{C}\right)\right)$.

(ii) If we consider the greatest and the least fuzzy implications, then

$$
I_{I_{G t}-I_{L t}}(x, y)= \begin{cases}1 & \text { if } x=0 \text { or } y=1, \\ 0 & \text { if } x=1 \text { and } y=0, \\ e & \text { otherwise, }\end{cases}
$$

is an e-generated implication that satisfies $(C P(N))$ for any negation $N$ such that $N(x) \neq$ 0,1 for all $x \in(0,1)$.

If $(\mathrm{CP}(\mathrm{N}))$ holds for an $e$-generated implication, then some necessary conditions must be satisfied as it is stated in the following proposition.

Proposition 20 Let $I_{1}, I_{2}$ be two implications and $N$ a fuzzy negation. If $I_{I_{1}-I_{2}}$ satisfies $(C P(N))$, then

(i) $0<N(x)<1$ if $x \in(0, e]$. Furthermore, if $N$ is continuous then $0<N(x)$ for all $0<x<1$.

(ii) $I_{1}(x, y)=1$ for all $x \leq N(e)$ and $y \in[0,1]$.

Note that for some specific forms of negations $N$ we can fully determine the initial implications needed to obtain an e-generated implication satisfying $(\mathrm{CP}(\mathrm{N}))$.

Proposition 21 Let $N$ be the following negation

$$
N(x)= \begin{cases}1 & \text { if } x=0 \\ e & \text { if } 0<x<1 \\ 0 & \text { if } x=1\end{cases}
$$

and $I_{1}, I_{2}$ two implications. Then

$I_{I_{1}-I_{2}}$ satisfies $(C P(N)) \Leftrightarrow I_{1}=I_{G t}$ and $I_{2}=I_{L t}$.

Proposition 22 Let $N$ be the following negation

$$
N(x)= \begin{cases}1 & \text { if } x=0 \\ e & \text { if } 0<x \leq e, \\ 0 & \text { if } x>e\end{cases}
$$

and $I_{1}, I_{2}$ two implications. Then $I_{I_{1}-I_{2}}$ satisfies $(C P(N))$ if and only if

$$
I_{1}(x, y)= \begin{cases}0 & \text { if } x>e \text { and } y=0 \\ 1 & \text { otherwise }\end{cases}
$$

and

$$
I_{2}(x, y)= \begin{cases}N^{\prime}(x) & \text { if } y=0, \\ 1 & \text { otherwise, }\end{cases}
$$

where $N^{\prime}$ is any fuzzy negation.
Now, we want to study when the contrapositive symmetry of the initial given implications is preserved by the e-generation method. Assuming that the negation $N$ satisfies some initial requirements, the first result gives us necessary and sufficient conditions for which the e-generated implications satisfy $(\mathrm{CP}(\mathrm{N}))$.

Proposition 23 Let $I_{1}$ and $I_{2}$ be two implications and $N$ a fuzzy negation such that $N(x)=e \Leftrightarrow x=e$ and $0<N(x)<1$ if $x \in(0,1)$. Then $I_{I_{1}-I_{2}}$ satisfies $(C P(N))$ if and only if $I_{1}(x, y)=1$ for all $x \leq e$ and

$$
I_{1}\left(x, \frac{y}{e}\right)=I_{1}\left(N(y), \frac{N(x)}{e}\right)
$$

when $e<x \leq 1$ and $0 \leq y \leq e$, and $I_{2}(x, y)=$ 0 for all $x \geq e$ and

$$
I_{2}\left(x, \frac{y-e}{1-e}\right)=I_{2}\left(N(y), \frac{N(x)-e}{1-e}\right)
$$

when $0 \leq x<e$ and $e<y \leq 1$.

In order to characterize the implications that satisfy Equations (1) and (2), let us start with a new construction method of a fuzzy implication from an initial one and a negation $N$.

Lemma 24 Let I be a fuzzy implication and $N$ a fuzzy negation.

(i) If $N(x) \in[0, e]$ for all $e<x \leq 1$ then

$$
I_{I, N}^{1}(x, y)= \begin{cases}1 & \text { if } x \leq e \\ I\left(N\left(\frac{N(x)}{e}\right), y\right) & \text { if } e<x \leq 1\end{cases}
$$

is a fuzzy implication.

(ii) If $N(x) \in[e, 1]$ for all $x<e$ then

$I_{I, N}^{0}(x, y)= \begin{cases}1 & \text { if } y=1, \\ 0 & \text { if } x \geq e, y<1, \\ I\left(N\left(\frac{N(x)-e}{1-e}\right), y\right) & \text { if } x<e\end{cases}$

is a fuzzy implication.

Lemma 25 Let I be a fuzzy implication and $N$ a strong negation with $N(e)=e$. Then

(i) I satisfies $(C P(N)) \Leftrightarrow$ $I_{I, N}^{1}\left(x, \frac{y}{e}\right)=I_{I, N}^{1}\left(N(y), \frac{N(x)}{e}\right)$ for all $e<x \leq 1$ and $0 \leq y \leq e$.

(ii) If I satisfies $(C P(N))$ then $I_{I, N}^{0}\left(x, \frac{y-e}{1-e}\right)=I_{I, N}^{0}\left(N(y), \frac{N(x)-e}{1-e}\right)$ for all $0<$ $x<e$ and $e<y \leq 1$. Moreover, if $I(x, 0)=$ $I(1, N(x))$ for all $x \in[0,1]$, the reciprocal holds too.

These lemmas are essential in order to give some partial answer to the preservation of the contrapositive symmetry. 
Proposition 26 Let $I_{1}$ and $I_{2}$ be two fuzzy implications and $N$ a strong fuzzy negation with fixed point $e$. Then the e-generated implication $I_{I_{I_{1}, N}^{1}-I_{I_{2}, N}^{0}}$ satisfies $C P(N)$ if and only if $I_{1}$ satisfies $(C P(N))$ and $I_{2}$ satisfy $(C P(N))$ except maybe when $y=0$.

Example 27 Consider $N=N_{C}, e=\frac{1}{2}$ and $I_{1}=I_{2}=I_{R S}$, the Rescher implication. Thus, we can construct $I_{I_{R S}, N_{C}}^{1}$ and $I_{I_{R S}, N_{C}}^{0}$ (see Figure 4 ) and the corresponding e-generated implication $I_{I_{I_{R S}, N_{C}}^{1}-I_{I_{R S}, N_{C}}^{0}}(x, y)=$

$= \begin{cases}0 & \text { if } x>\frac{1}{2} \text { and } y<x-\frac{1}{2} \\ 1 & \text { if } x=0 \text { or } y=1 \text { or }\left(\frac{1}{2}<y<1, x \leq y-\frac{1}{2}\right), \\ \frac{1}{2} \quad \text { otherwise. }\end{cases}$

Since $I_{R S}$ satisfies $\left(C P\left(N_{C}\right)\right), \quad I_{I_{I_{R S}, N_{C}}^{1}}-I_{I_{R S}, N_{C}}^{0}$ (see Figure 5) satisfies $\left(C P\left(N_{C}\right)\right)$.

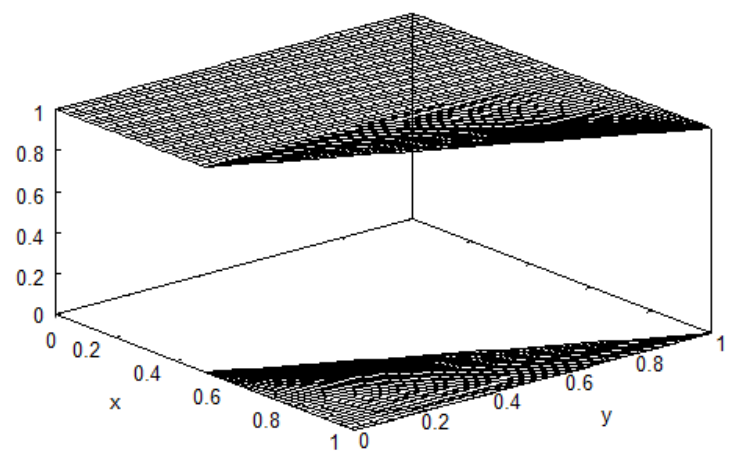

(a) $I_{I_{R S}, N_{C}}^{1}$ with $e=\frac{1}{2}$

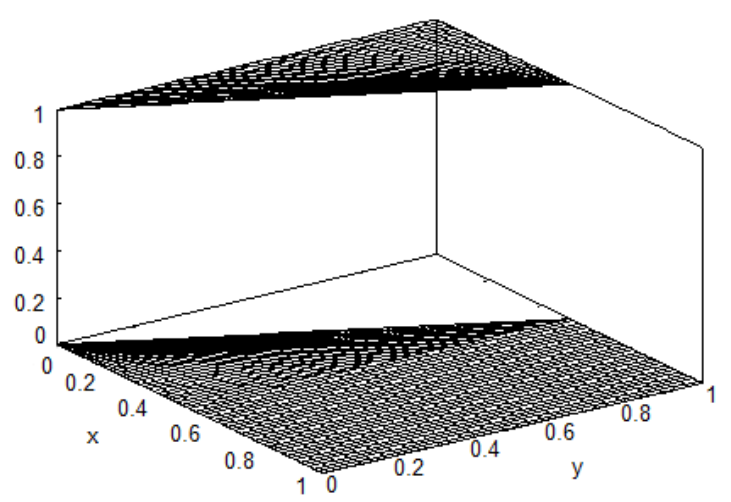

(b) $I_{I_{R S}, N_{C}}^{0}$ with $e=\frac{1}{2}$

Figure 4: Plots of the constructed implications by Lemma 24 from $I_{R S}$ and $N_{C}$

\section{Exchange Principle and the Weak Law of Importation}

In this section, the main goal is to study the preservation of the exchange principle and of the weak law of importation. As it has already noted, the exchange principle is not preserved by majority of construction methods of a fuzzy implication from two given ones (see Remarks 6.1.5. and 6.2.5. in

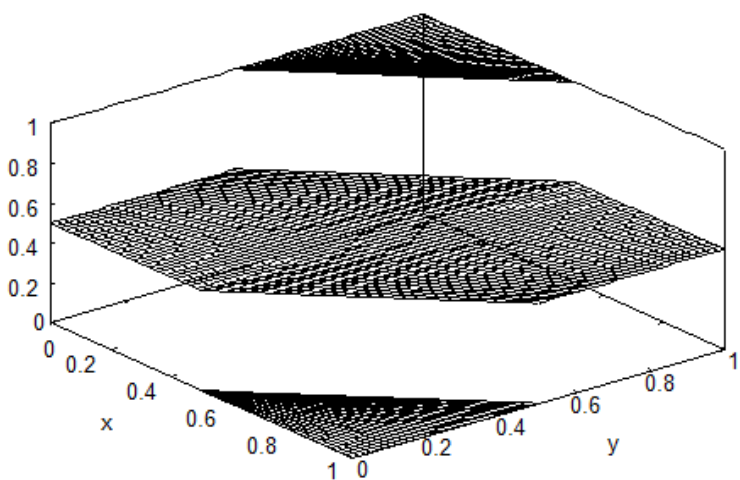

Figure 5: Plot of the e-generated implication $I_{I_{I_{R S}, N_{C}}^{1}}-I_{I_{R S}, N_{C}}^{0}$

[5]). Note that the conjugacy method preserves always the exchange principle. However, the conjugacy method is a construction method of a fuzzy implication from another given one. The following example shows that it is not sufficient that the two initial implications satisfy (EP) to obtain an e-generated implication satisfying (EP).

Example 28 Let $I_{G D}$ be the Gödel implication and $I_{2}(x, y)=I_{U_{L K}, N_{C}}(x, y)=$

$= \begin{cases}\max \{y-x+0.5,0\} & \text { if } \max \{1-x, y\} \leq 0.5, \\ \min \{y-x+0.5,1\} & \text { if } \min \{1-x, y\}>0.5, \\ I_{K D}(x, y) & \text { otherwise. }\end{cases}$

Both implications satisfy (EP) because $I_{G D}$ is an $R$-implication and $I_{2}$ is a $(U, N)$-implication where $U_{L K}$ is the disjunctive uninorm from the class $U_{\max }$ generated by the triple $\left(T_{L K}, S_{L K}, 0.5\right)$ (see Table 2.6. and Example 5.3.6. in [5]). However, $I_{I_{G D}-I_{U_{L K}, N_{C}}}(x, y)=$

$$
= \begin{cases}1 & \text { if } x=0, \\ y & \text { if } x>0, y \leq \frac{1}{2}, x>2 y, \\ \frac{1}{2} & \text { if } x>0, y \leq \frac{1}{2}, x \leq 2 y, \\ \max \left\{y-\frac{x}{2}+\frac{1}{4}, \frac{1}{2}\right\} & \text { if } x>0, y>\frac{1}{2}, \\ & \max \{1-x, 2 y-1\} \leq \frac{1}{2}, \\ \min \left\{y-\frac{x}{2}+\frac{1}{4}, 1\right\} & \text { if } x>0, y>\frac{1}{2}, \\ & \min \{1-x, 2 y-1\}>\frac{1}{2} \\ \frac{1}{2}+\frac{1}{2} I_{K D}(x, 2 y-1) & \text { otherwise, }\end{cases}
$$

(see Figure 6) does not satisfy (EP), just take $x=$ $0.1, y=0.8$ and $z=0.6$.

The problem in the previous example is that $I_{U_{L K}, N_{C}}(x, y)=0$ for some $x, y>0$. Thus, not allowing this case (EP) is almost preserved.

Proposition 29 Let $I_{1}, I_{2}$ be two implications such that $I_{2}(x, y)>0$ if $y>0$. Then $I_{I_{1}-I_{2}}$ satisfies (EP) if and only if $I_{1}$ satisfies (EP) and

$$
I_{2}\left(x, I_{2}(y, z)\right)=I_{2}\left(y, I_{2}(x, z)\right)
$$

if $x=0$ or $y=0$ or $(x, y, z>0)$. 


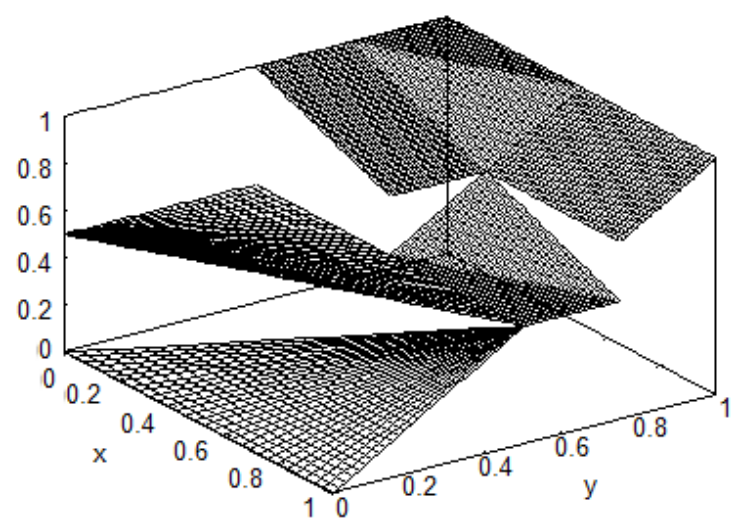

Figure 6: Plot of the e-generated implication $I_{I_{G D}-I_{U_{L K}, N_{C}}}$

Remark 30 1. If the initial implications $I_{1}$ and $I_{2}$ satisfy $(E P)$ and $I_{2}(x, y)>0$ if $y>0$ then their e-generated implication satisfies also (EP).

2. There exist e-generated implications satisfying (EP) such that their initial implication $I_{2}$ does not need to satisfy (EP) (see Example 31).

Example 31 Consider the Weber implication $I_{W B}$ and

$$
I_{2}(x, y)= \begin{cases}y & \text { if } x=1 \\ 1-x & \text { if } x \leq \frac{1}{2}, y=0 \\ 0 & \text { if } x>\frac{1}{2}, y=0 \\ 1 & \text { otherwise. }\end{cases}
$$

It is well-known that $I_{W B}$ satisfies (EP). However $I_{2}$ does not satisfy (EP), just consider $x=0.6, y=$ 0.4 and $z=0$. Even in these conditions their $e$ generated implication $I_{I_{W B}-I_{2}}(x, y)=$

$$
= \begin{cases}1 & \text { if } x=0 \text { or }(0<x<1, y>e) \\ e & \text { if } 0<x<1, y \leq e \\ y & \text { if } x=1\end{cases}
$$

satisfies (EP), just applying Proposition 29.

Note that Equation (3) is almost the exchange principle except for the case $x, y>0$ and $z=0$. So, the following result is immediate.

Corollary 32 Let $I_{1}, I_{2}$ be two implications such that $I_{2}(x, y)>0$ if $y>0$ and $I_{2}$ satisfies the right contraposition with respect to its natural negation, that is $I_{2}\left(x, N_{I_{2}}(y)\right)=I_{2}\left(y, N_{I_{2}}(x)\right)$. Then $I_{I_{1}-I_{2}}$ satisfies (EP) if and only if $I_{1}$ and $I_{2}$ satisfy (EP).

From now on, we focus our attention to the law of importation. Recently, the law of importation has been extensively studied (see [15], [16], [17]). In addition to their satisfaction for the most common classes of implications, even its relationship with other properties of fuzzy implications like (EP) and (WLI) has been established (see [14]). The results obtained for this property (in fact, for the weak law of importation) are quite similar to the ones of the exchange principle.

Lemma 33 Let $I_{1}$ and $I_{2}$ be two implications. Then if $I_{I_{1}-I_{2}}$ satisfies (WLI) with a function $F$, then $F$ is positive, i.e., $F(x, y)>0$ when $x, y>0$.

However, given two implications that satisfy (WLI) with a positive function $F$, their e-generated implication does not need to satisfy (WLI) with $F$ as the following example shows.

Example 34 Consider the following implication twice $I_{U, N_{C}}(x, y)=$

$$
= \begin{cases}0 & \text { if } x>\frac{1}{2} \text { and } y<\frac{1}{2} \\ 1-x & \text { if } y=\frac{1}{2} \\ y & \text { if } x=\frac{1}{2} \\ \max \{1-x, y\} & \text { otherwise }\end{cases}
$$

where $U$ is the disjunctive uninorm from the class $U_{\max }$ generated by the triple $\left(T_{D}, S_{M}, 0.5\right)$. This implication satisfy (WLI) (in fact, $\left(L I_{U}\right)$, i.e., (LI) with a uninorm) with the positive uninorm $U_{N_{C}}(x, y)=N_{C}\left(U\left(N_{C}(x), N_{C}(y)\right)\right)$ because it is a $(U, N)$-implication from $U$ and $N=N_{C}$ (see [17]). However, the corresponding e-generated implication $I_{I_{U, N_{C}}-I_{U, N_{C}}}(x, y)=$

$$
= \begin{cases}0 & \text { if } x>\frac{1}{2}, y<\frac{1}{4}, \\ \frac{1}{2}-\frac{x}{2} & \text { if } x>\frac{1}{2}, y=\frac{1}{4}, \\ y & \text { if } x=\frac{1}{2}, \\ \max \left\{\frac{1}{2}-\frac{x}{2}, y\right\} & \text { if }\left(0<x<\frac{1}{2}, y \leq \frac{1}{2}\right) \text { or } \\ & \left(x>\frac{1}{2}, \frac{1}{4}<y \leq \frac{1}{2}\right), \\ \frac{1}{2} & \text { if } x>\frac{1}{2}, \frac{1}{2}<y<\frac{3}{4}, \\ 1-\frac{x}{2} & \text { if } x>\frac{1}{2}, y=\frac{3}{4}, \\ \max \left\{1-\frac{x}{2}, y\right\} & \text { if }\left(0<x<\frac{1}{2}, y>\frac{1}{2}\right) \text { or } \\ & \left(x>\frac{1}{2}, y>\frac{3}{4}\right), \\ 1 & \text { if } x=0,\end{cases}
$$

(see Figure 7) does not satisfy (WLI) with $U_{N_{C}}$, just take $x=0.2, y=0.6$ and $z=0.7$.

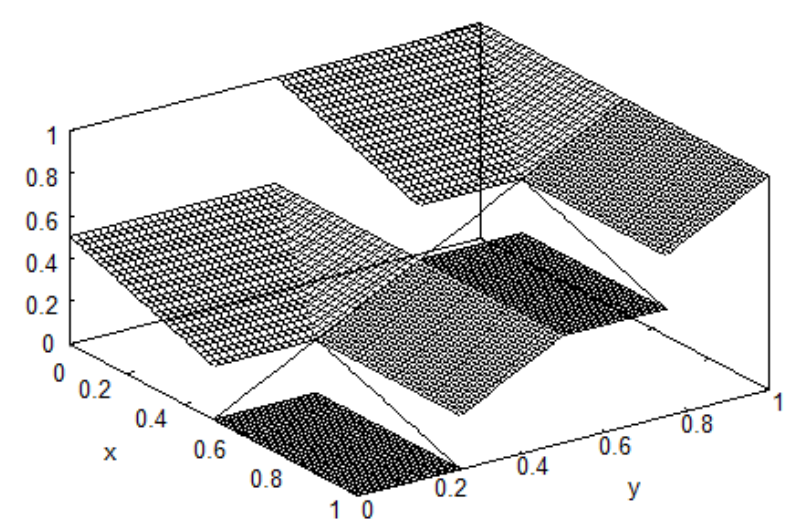

Figure 7: Plot of the e-generated implication $I_{I_{U, N_{C}}-I_{U, N_{C}}}$ 
Proposition 35 Let $I_{1}$ and $I_{2}$ be two implications such that $I_{2}(x, y)>0$ if $y>0$ and let $F$ be a commutative, non-decreasing and positive binary function. Then $I_{I_{1}-I_{2}}$ satisfies (WLI) with $F$ if and only if $I_{1}$ satisfies (WLI) with $F$ and

$$
I_{2}(F(x, y), z)=I_{2}\left(x, I_{2}(y, z)\right)
$$

if $x=0$ or $y=0$ or $(x, y, z>0)$.

Remark 36 If the initial implications $I_{1}$ and $I_{2}$ satisfy (WLI) with a positive function $F$ and $I_{2}(x, y)>0$ if $y>0$ then their e-generated implication satisfies (WLI) with the same function $F$.

Note that Equation (4) is almost the weak law of importation except when $x, y>0$ and $z=0$. Thus, the following result is immediate.

Corollary 37 Let $I_{1}$ and $I_{2}$ be two implications such that $I_{2}(x, y)>0$ if $y>0$ and let $F$ be a commutative, non-decreasing and positive binary function such that $N_{I_{2}}(F(x, y))=I_{2}\left(x, N_{I_{2}}(y)\right)$. Then $I_{I_{1}-I_{2}}$ satisfies (WLI) with $F$ if and only if $I_{1}$ and $I_{2}$ satisfy (WLI) with $F$.

\section{Conclusions and Future Work}

In this paper, a new generation method of a fuzzy implication from two previous ones is introduced and the propagation of the basic properties from the initial implications to the e-generated implication is studied. The trademark of this method is the control of the increasingness of the generated implication on the second variable, that can be of interest in fuzzy control. Another important advantage of the method presented in this paper is the preservation of the exchange principle and the law of importation, under some minimal conditions, to the generated implication.

However, the behaviour of some properties, such as the contrapositive symmetry, is an open question since we only know some special cases. In addition, it is worth to study the preservation of the distributivity properties to the e-generated implication. Finally, note that as a future work the same construction method but now on the first variable is possible as well as some kind of ordinal sum of implication functions combining the method introduced here and the one corresponding to the first variable.

\section{Acknowledgment}

This paper has been partially supported by the Spanish Grant MTM2009-10320 with FEDER support.

\section{References}

[1] S. Gottwald. A Treatise on Many-Valued Logic. Research Studies Press, Baldock, 2001.
[2] E.E. Kerre, C. Huang, and D. Ruan. Fuzzy Set Theory and Approximate Reasoning. Wu Han University Press, Wu Chang, 2004.

[3] M. Mas, M. Monserrat, J. Torrens, and E. Trillas. A survey on fuzzy implication functions. IEEE Transactions on Fuzzy Systems, 15(6):1107-1121, 2007.

[4] M. Baczyński and B. Jayaram. (S,N)- and Rimplications: A state-of-the-art survey. Fuzzy Sets and Systems, 159:1836-1859, 2008.

[5] M. Baczyński and B. Jayaram. Fuzzy Implications. Springer, Berlin Heidelberg, 2008.

[6] H. Bustince, V. Mohedano, E. Barrenechea, and M. Pagola. Definition and construction of fuzzy DI-subsethood measures. Information Sciences, 176:3190-3231, 2006.

[7] H. Bustince, M. Pagola, and E. Barrenechea. Construction of fuzzy indices from fuzzy DIsubsethood measures: application to the global comparison of images. Information Sciences, 177:906-929, 2007.

[8] P. Yan and G. Chen. Discovering a cover set of ARsi with hierarchy from quantitative databases. Information Sciences, 173:319-336, 2005.

[9] B. Jayaram and R. Mesiar. I-fuzzy equivalence relations and i-fuzzy partitions. Inf. Sci., 179(9):1278-1297, 2009.

[10] E. Trillas, M. Mas, M. Monserrat, and J. Torrens. On the representation of fuzzy rules. Int. J. Approx. Reasoning, 48(2):583-597, 2008.

[11] S. Massanet and J. Torrens. On a new class of fuzzy implications: h-implications and generalizations. Information Sciences, 181(11):2111 2127, 2011.

[12] E.P. Klement, R. Mesiar, and E. Pap. Triangular norms. Kluwer Academic Publishers, Dordrecht, 2000.

[13] J.C. Fodor and M. Roubens. Fuzzy Preference Modelling and Multicriteria Decision Support. Kluwer Academic Publishers, Dordrecht, 1994.

[14] S. Massanet and J. Torrens. The law of importation versus the exchange principle on fuzzy implications. Fuzzy Sets and Systems, 168(1):47 - 69, 2011.

[15] B. Jayaram. On the law of importation $(x \wedge$ $y) \rightarrow z \equiv(x \rightarrow(y \rightarrow z))$ in fuzzy logic. IEEE Transactions on Fuzzy Systems, 16:130$144,2008$.

[16] M. Mas, M. Monserrat, and J. Torrens. The law of importation for discrete implications. Information Sciences, 179:4208-4218, 2009.

[17] M. Mas, M. Monserrat, and J. Torrens. A characterization of $(\mathrm{U}, \mathrm{N}), \mathrm{RU}, \mathrm{QL}$ and $\mathrm{D}$ implications derived from uninorms satisfying the law of importation. Fuzzy Sets and Systems, 161:1369-1387, 2010. 